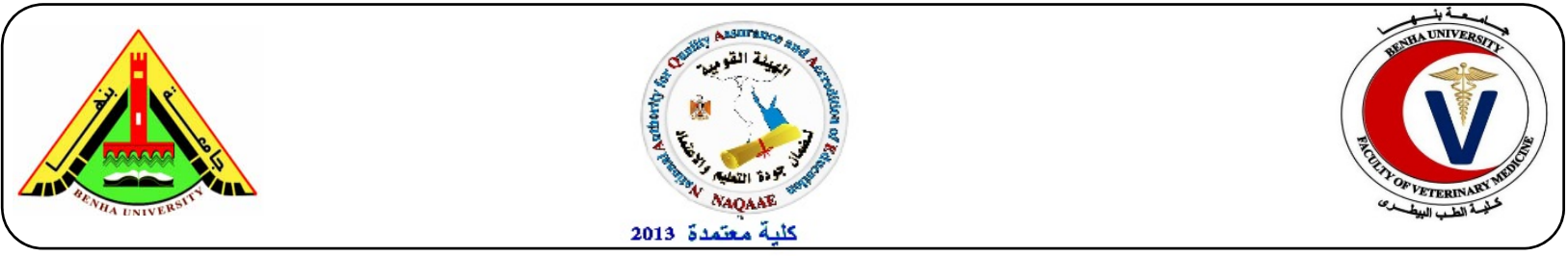

\title{
Impact of probiotic and prebiotic on the lysozyme response of broilers against clostridial infection.
}

\author{
Ashraf A. Abd El Tawab ${ }^{1}$, Ahmed M. Amar ${ }^{2}$, Mamdouh A. Elshorbagy ${ }^{3}$, Ehab W. Mostafa ${ }^{4}$ \\ ${ }^{1}$ Department of Bacteriology, Mycology and Immunology, Faculty of Veterinary Medicine, Benha \\ University. ${ }^{2}$ Department of Microbiology, Faculty of Veterinary Medicine, Zagazig University. ${ }^{3}$ \\ Research unite Animal Health Research Institute El-Doki. ${ }^{4}$ Veterinary Medicine Directorate Sharqia.
}

\section{A B S T R A C T}

A total of 75 one-day old chicks (Cobb local breed) At the 1 day old the chicks were randomly divided into 3 equal separate groups; 25 chicks each. Group 1 fed on prebiotic beta glucan $200 \mathrm{gram} /$ ton, group 2 fed on probiotic saccharomyces servicee 500gram/ton and group 3 control fed on balanced commercial ration. The result revealed that the group of broiler fed on prebiotic increase the lysozyme more than the group of broiler fed on probiotic and after artificial infection, the group treated with prebiotic was able to face the clostridial infection more than group treated with probiotic. The histopathological examination showed that increase length and width of intestinal villi of chicken treated with prebiotic more than that of probiotic.

Keywords: Prebiotic, Probiotic, Clostridium, Broilers, Intestinal Villi, Lysozyme.

(http://www.bvmj.bu.edu.eg)

(BVMJ-28(2): 208-213, 2015)

\section{INTRODUCTION}

$\mathrm{T}$ The most widely used bacteria as probiotics are the lactobacilli and bifid bacteria but products incorporating other organisms such as Gram positive cocci, bacilli, yeasts and E.coli have also been applied Holzapfel and Schillinger, (2002). Consist of single or a combination of bacteria (mainly lactic acid producing) or yeasts. Bacteria and yeasts may be included as spores or as living microorganisms. Most probiotic products available on the market contain Lactobacilli, Bacilli, Streptococci, Bifidobacterii and Sacharomcyces varieties. Probiotics act by competitive exclusion, lower gut $\mathrm{pH}$, produce bacteriocins, lysozyme and peroxides, and stimulate the immune system. The combined application of prebiotics and probiotics is called synbiotics (Panda et al., (2006). The concept of prebiotics was defined as "a nondigestible food ingredient that beneficially affects the host by selectively stimulating the growth and/or activity of one or a limited number of bacteria species in the colon, and thus improves host health" (Gibson and Roberfroid 1995). Food ingredients that are classified as prebiotics have to be resistant to absorption by the GIT, be fermented by intestinal organisms, and selectively stimulate beneficial bacteria associated with animal health (Roberfroid 2007). Only inulin and trans-galacto oligosaccharides are currently as recognized meeting all these criteria (Roberfroid 2007. Prebiotics can increase digestion of nutrients in the feed by increasing the length and width of intestinal villi Sinovec and Markovic (2005). Tasco ${ }^{\circledR}$ which is a product made from sun-dried brown seaweed has been reported to have prebiotic effects when added to chicken diets. Wiseman (2012) demonstrated that feeding this product improves intestinal 
microstructure in broilers. Prebiotics are defined as food ingredients that stimulate selectively the growth and activity of beneficial microorganisms such as Bifidobacteria and Lactobacillus in the gut and thereby benefit health, can reduce the numbers of clostridia and increase colonization resistance to pathogens Cummings and MacFarlane, (2002). Lysozyme is anatural antimicrobial protein, occurs in a number of animal secretions. It is considered an important component of the innate immune system. It exerts bacteriolytic activity by hydrolysing the b1,4-glycosidic linkage between Nacetlymuraminic acid and $\mathrm{N}$-acetyl glucosamine of bacterial cell wall peptidoglycan and is most effective against many Gram-positive bacteria Phillips, D.C. (1996). Zhang et al., (2006) reported the antimicrobial activity of lysozyme against isolates of $C$. perfringens type A associated with broiler NE in vitro, and considered that lysozyme could kill the bacterial pathogen, lysozyme in vitro could not only kill $\mathrm{C}$. perfringens, but could also reduce production of a-toxin, the causative agent for intestinal lesions. MOS supplementation resulted in significant improvement in antibody responses in broiler and layers (Cotter et al., 2000; Raju and Devegowda, 2002). Pelicano et al., (2005) demonstrated that supplementation with probiotics, prebiotics, organic acids or the combination of each benefits the small intestine histological indexes. These measurements include villi height and crypt depth for 21day-old broiler chickens. Other ingredients such as lysozyme, lactoferrin and antibiotics have increased the villi height in the small intestine, and subsequently improved the nutrient absorption by broiler chickens.

\section{MATERIAL AND METHODS}

\subsection{Experimental birds}

The used chicks (75 one day old chicks) were floor reared and fed commercial balanced ration. At the 1 day old of age the chicks were randomly divided into 3 equal separate groups; 25 chicks each. Group 1 fed on prebiotic beta glucan 200gram/ton. Group 2 fed on probiotic saccharomyces servicee $500 \mathrm{gram} / \mathrm{ton}$. Group 3 control fed on balanced commercial ration. At age of 22 days birds of groups 1, 2 were orally inoculated with overnight culture of clostridium perfringes109 colony, while birds of group 1 were left as negative control. All groups were subjected to daily observation for clinical signs and/or mortalities. Blood sample were collected a day before inoculation of random 5 chicken from each group and mark them and collect blood sample from those marked chicken twice $24 \mathrm{hr}$ after inoculation and $72 \mathrm{hr}$ after inoculation. Serum was separated from blood collected without anticoagulant and used for measurement lysozyme activity. One bird /group was randomly sacrificed at day 27 for postmortem examination with collection of tissue samples for histopathological examination. Obtained results are shown in tables.

\subsection{Detection of lysozyme activity:}

Lysoplates were prepared by dissolving $0.1 \%$ agarose in $0.06 \mathrm{M}$ of PBS at $\mathrm{pH} 6.3$, $500 \mathrm{mg}$ of Micrococcus lysodeikticus in 5 $\mathrm{ml}$ saline were added to 1 litter of agarose. In lysoplates, $25 \mu \mathrm{l}$ of serum samples and standard lysozyme were put in each well. After 18 hours, the diameter of cleared zones was measured for both standard lysozyme and serum sample and the concentration was estimated. Schltz (1987)

\section{RESULTS}

\subsection{Detection of lysozyme in artificially infected broilers:}

Seventy five-one day old chicken classified into 3 groups (1, 2 and 3). Group 1 fed on prebiotic beta glucan, group 2 fed on probiotic saccharomyces servicee and group 3 control fed on balanced commercial ration. An overnight culture of clostridium was prepared containing $10^{9}$ 
microorganism $/ \mathrm{ml}$. At one day (day 22) before inoculation blood samples were collected from 5 random chickens from each group and left to be coagulated. Inoculation was done at day 23. Another blood sample at day 24 was collected from each bird. The last blood sample was collected after 72 hours from inoculation at day 27. The results were shown in figures 14. Figure 1 shows the concentration of lysozyme in free ration fed broilers, it is observed reduction of lysozyme content especially after $C$. perfringens experimental infection. Figure 2 shows the effects of probiotic on lysozyme concentration in broilers experimentally infected with $C$. perfringens, it declares the recovery of lysozyme on the $3^{\text {rd }}$ day post-infection. Figure 3 shows the effects of prebiotic on lysozyme concentration in broilers experimentally infected with $C$. perfringens; it declares the recovery of lysozyme on the $3^{\text {rd }}$ day post-infection. Figure 4 shows the comparison of the effects of probiotic and prebiotic with freeration fed broilers experimentally infected with C. perfringens.

\subsection{Histopathological changes of artificial infected group treated with probiotic and prebiotic:}

The group treated with probiotic and prebiotic showed the following histopathological changes: 1. Liver showed focal leukocytic infiltration in the fibrous connective tissue (CT) of the portal area, and in hepatic parenchyma, proliferation of van kupffer cells lining hepatic sinusoids (figure $5 \& 6$ ). 2- Intestine showed marked elongation of intestinal villi and vacuolation of the goblet cells (figure $7 \& 8$ ).

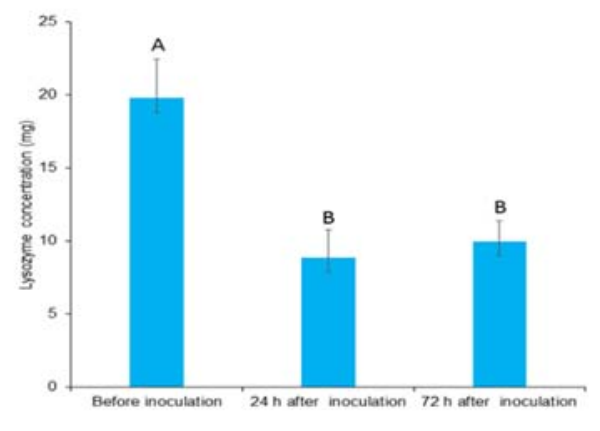

Figure 1. Effects of experimental infection with C. perfringens on lysozyme formation in free-ration fed-broilers.

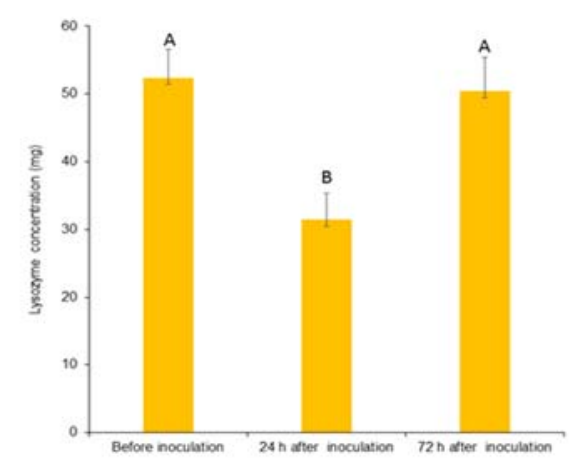

Figure 2. Effects of experimental infection with $C$. perfringens on lysozyme formation in probiotic fed broilers

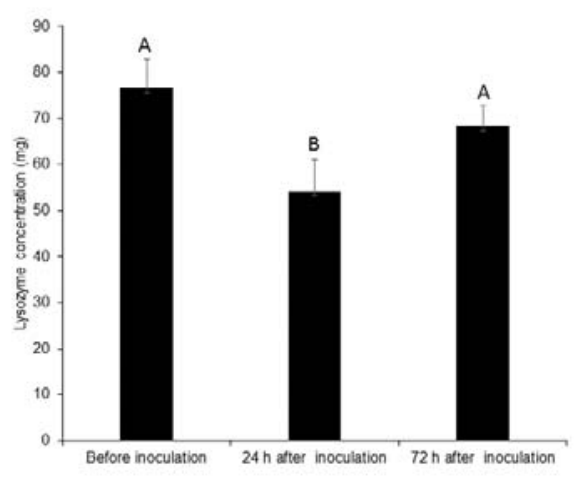

Figure 3. Effects of experimental infection with C. perfringens on lysozyme formation in prebiotic fed-broilers 

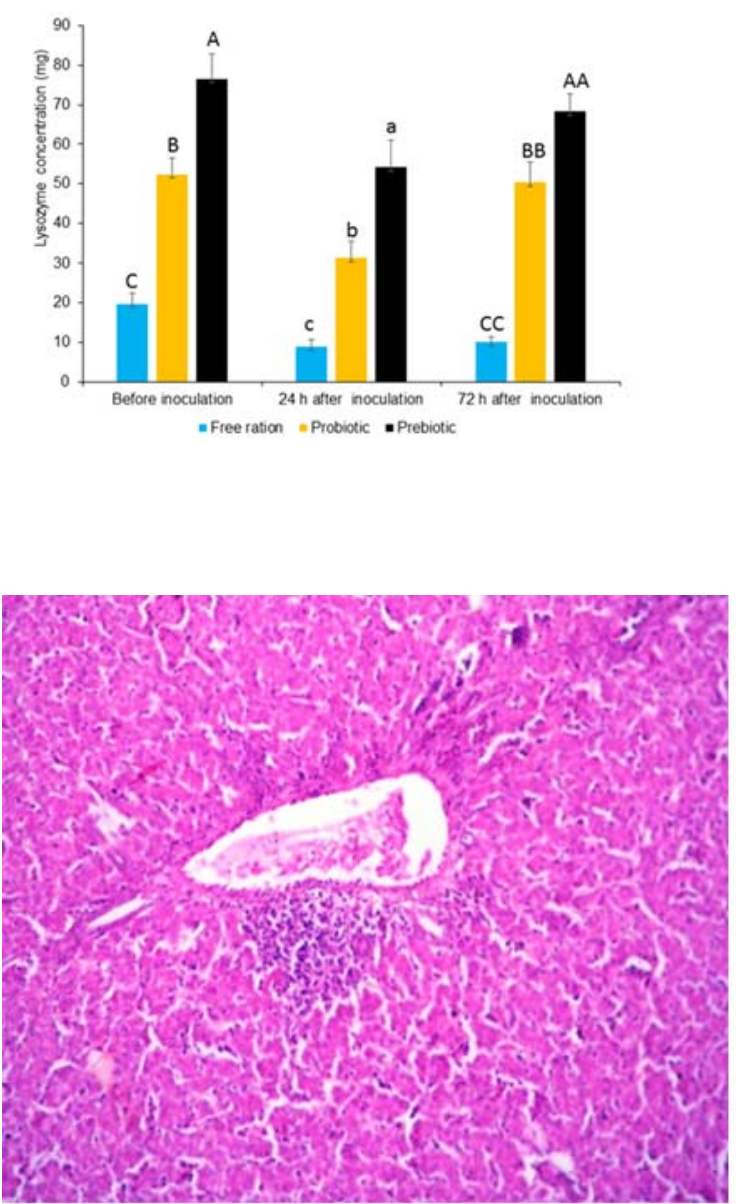

fig. 5

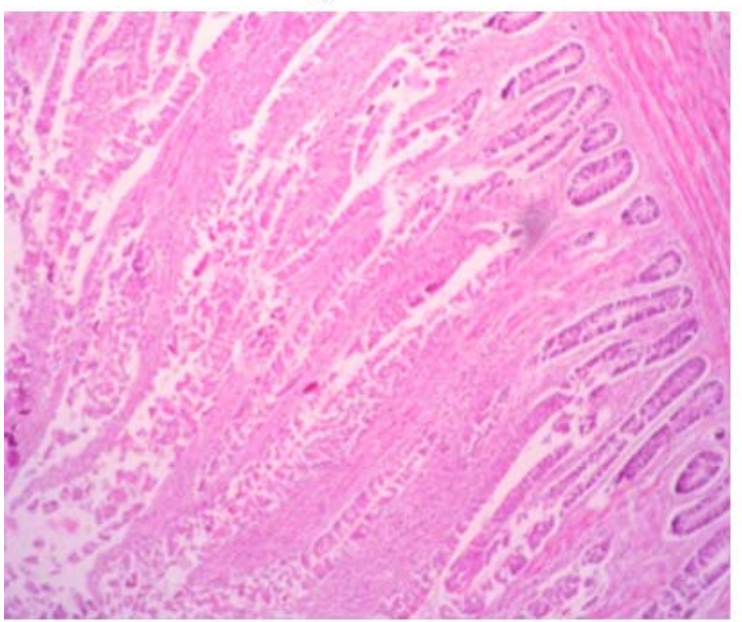

fig. 7
Figure 4. Comparison of the effects of experimental infection with C. perfringens on lysozyme formation in free ration, probiotic and prebiotic fed-broilers

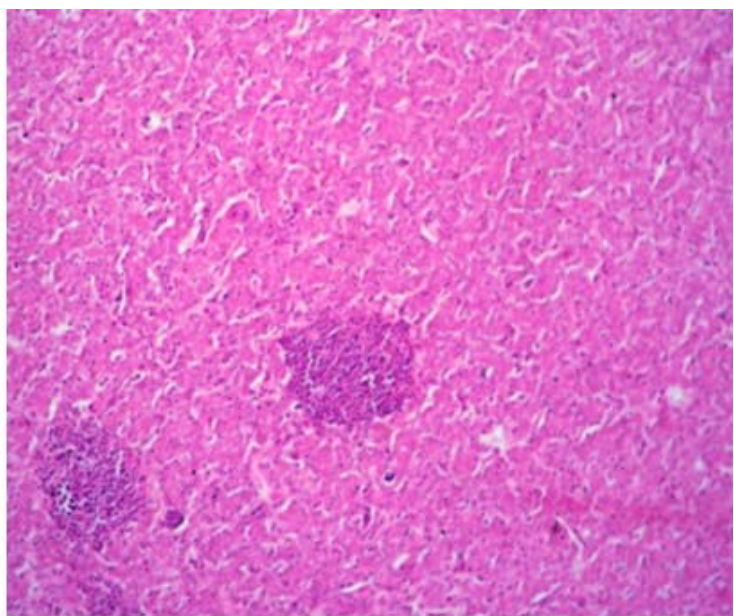

fig. 6

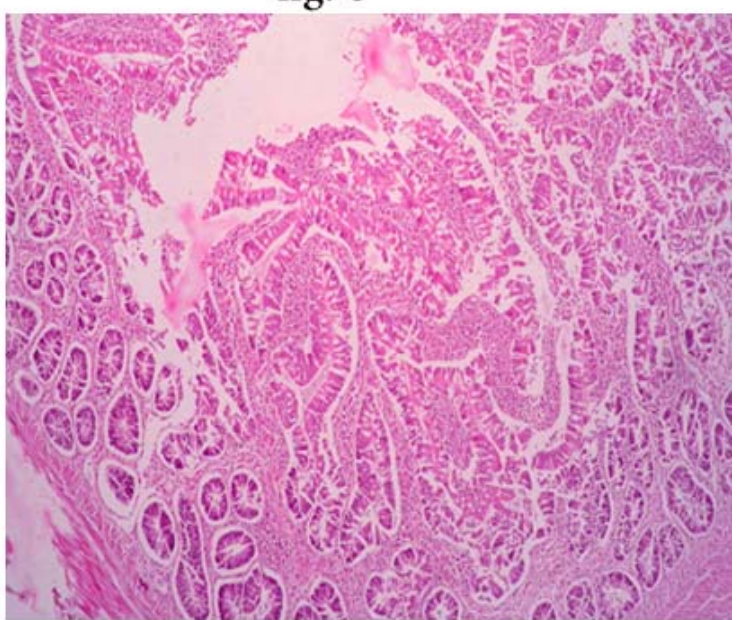

fig. 8

Figure 5. Liver treated with prebiotic show leukocytic cell infiltration. Figure 6. Liver illustrating multifocal leukocytic cell infiltration in hepatic parenchyma. Figure 7. Intestine shows increased highest of intestinal villi and vacuolation of goblet cells. Figure 8. Intestine by higher magnification of one of intestinal villi demonstrating hyperplastic proliferation of the erythrocytes and vacuolation of the goblet cells.

\section{DISCUSSION}

In this study, seventy five-one day old chicken classified into 3 groups (1,2 and 3$)$.
Group 1 fed on prebiotic beta glucan, group 2 fed on probiotic saccharomyces servicee and group 3 control fed on balanced commercial ration. An overnight culture of 
clostridium was administered orally, blood samples were collected, lysozyme concentrations were measures and histopathological examination was performed. Figure 1 showed that the concentration of lysozyme in free ration fed broilers, was a significantly reduced $(p<$ 0.05 ) in lysozyme content especially after experimental induction of $C$. perfringens. However, the effect of probiotic on lysozyme concentration in broilers experimentally infected with $C$. perfringens was clear, the concentration of lysozymes was higher than the free ration fed broilers, the recovery of lysozyme on the $3^{\text {rd }}$ day post-infection was very clear (Figures $2 \&$ 4). Figure 3 shows the effects of prebiotic on lysozyme concentration in broilers experimentally infected with $C$. perfringens; it declares the recovery of lysozyme on the $3^{\text {rd }}$ day post-infection. Figure 4 shows the comparison of the effects of probiotic and prebiotic with freeration fed broilers experimentally infected with $C$. perfringens. It is very clear the beneficial effects of using probiotics and prebiotics in induction of lysozyme formation and rapid recovery of lysozyme post-infection. In addition, prebiotic administration was very helpful in recovery of the histopathological changes and protection against $C$. perfringens adverse effects (figures 5-8). In agreement with our findings, Hofacre et al. (1998) showed that a commercial probiotic preparation reduced gross lesions of necrotic enteritis in chickens, but the protection was far less than that conferred by competitive exclusion flora. Lactobacilli were successful in decreasing mortality due to necrotic enteritis from $60 \%$ to $30 \%$ in an experimental challenge trial, when they were given orally at day 1 of life (Hofacre et al., (2003). In their experiment, feed conversion was decreased in the group that was given lactobacilli, while the weight gain was not altered. Regarding prebiotics, when fructooligosaccharides (FOS) were added to a continuous culture model of the human intestine or to batch cultures inoculated with human faeces in vitro, growth of $C$. perfringens was enhanced (Bello et al., (2001); McBain \& MacFarlane, 2001). In rats, cats and dogs it was shown, however, that addition of FOS to the diet resulted in significantly fewer $C$. Perfringens bacteria in the intestinal tract compared with animals fed unsupplemented diet (Gallaher et al., (1996); Sparkes et al., (1998); Swanson et al., (2002). Unlikely, Hofacre et al., (2003) showed that neither addition of FOS or mannooligosaccharides to the diet of broilers had a significant effect on mortality caused by necrotic enteritis, on weight gain and on feed conversion in 6week-old broilers, in an experimental challenge experiment.

\section{REFERENCES}

Bello, F.D., Walter, J., Hertel, C., Hammes, W.P. 2001. In vitro study of prebiotic properties of levan-type exopolysaccharides from Lactobacilli and non-digestible carbohydrates using denaturing gradient gel electrophoresis. Systematic and Applied Microbiology, 24:232-237.

Cotter, P.F, Malzone, A., Paluch, B., Lilburn, M.S., Sefton, A.E. 2000. Modulation of humoral immunity in commercial laying hens by a dietary prebiotic. Poult. Sci., 79(Suppl. 1): 38.

Cummings, J.H., McFarlane, G.T., 2002. Gastrointestinal effects of prebiotics. Br. J. Nutr., 87(2):145-151.

Gallaher, D.D., Stallings, H., Blessing, L.L., Busta, F.F., Brady, L.J. 1996. Probiotics, cecal microflora, and aberrant crypts in the rat colon. Journal of Nutrition, 126:1362-1371.

Gibson, G. R., Roberfroid, M. B. 1995. Dietary modulation of the human colonic microbiota: introducing the concept of prebiotics. Journal of Nutrition, 125:1401-1412.

Hofacre, C.L., Froyman, R., Gautrias, B., George, B., Goodwin, M.A., Brown, J. 1998. Use of Aviguard and other intestinal bioproducts in experimental Clostridium perfringens associated 
necrotizing enteritis in broiler chickens. Avian Diseases, 42:579584.

Hofacre, C.L., Beacorn, T., Collett, S., Mathis, G. 2003. Using competitive exclusion, mannan-oligosaccharide and other intestinal products to control necrotic enteritis. Journal of Applied Poultry Research, 12:60-64.

Holzapfel, W. H., Schillinger, U. 2002. Introduction to pre- and probiotics. Food Research International, 35:109116.

McBain, A.J., MacFarlane, G.T. 2001. Modulation of genotoxic enzyme activities by non-digestible oligosaccharide metabolism in invitro human gut bacterial ecosystems. Journal of Medical Microbiology, 50:833-842.

Panda, K., Rama, Rao, S.V., Raju, M.V.L.N., 2006. Natural growth promoters have potential in poultry feeding systems. Feed Tech. 10(8): 23-25

Pelicano, E R L., Souze, P.A. Souze, H B. A., Figueiredo, D F., Boiage, M M., Carvalho, S. R., Bordon, V. F. 2005. Intestinal musosa development in broiler chickens fed natural growth promoters. Braz. J. Poult. Sci. 7:221229.

Phillips, D.C. 1996 The three-dimensional structure of an enzyme molecule. Scientific American, 215:78-79.

Roberfroid, M. 2007. Prebiotics: the concept revisited. J. Nutr. 137:830837.

Raju, Mvln, Devegowda, G. 2002. Esterified-Glucomannan in broiler chicken diets-contaminated with aflatoxin, ochratoxin and T-2 toxin: Evaluation of its binding ability (in vitro) and efficacy as immunomodulator. Asian- Aust. J. Anim. Sci., 15:1051-1056.

Schltz, L.A., 1987. Methods in Clinical Chemistry. The CV Mosby, St Louis, Missouri, USA, pp. 742-746.
Sinovec, Z., Markovic, R.2005. Using prebiotics in poultry nutrition. Biotech. Anim. Husbandry 21:235239.

Sparkes, A.H., Papasouliotis, K., Sunvold, G., Werrett, G., Gruffydd-Jones, E.A., Egan, K., Gruffydd-Jones, T.J., Reinhart, G. 1998. Effect of dietary supplementation with fructooligosaccharides on faecal flora of healthy cats. American Journal of Veterinary Research, 59:436-440.

Swanson, K.S., Grieshop, C.M., Flickinger, E.A., Bauer, L.L., Chow, J., Wolf, B.W., Garleb, K.A., Fahey, G.C., Jr. 2002. Fructoologo-saccharides and Lactobacillus acidophilus modify gut microbial populations, total tract nutrient digestibilities and faecal protein catabolite concentrations in healthy adult dogs. Journal of Nutrition, 132:3721-3731

Wiseman, M. 2012. Evaluation of Tasco $\AA$ as a candidate prebiotic in broiler chickens. MSc. Thesis. Faculty of Agriculture, Dalhousie University.

Zhang, G., Darius, S., Smith, S.R., Ritchie, S.J. 2006. In vitro inhibitory effect of hen egg white lysozyme on Clostridium perfringens type A associated with broiler necrotic enteritis and its a-toxin production. Letters in Applied Microbiology, 42: 139-143. 